\title{
CLINICAL EXOME SEQUENCING IN DEMENTIAS: A PRELIMINARY STUDY
}

\author{
Bojan Zalar ${ }^{1}$, Aleš Maver ${ }^{2}$, Ana Kovanda ${ }^{2}$, Ana Peterlin² \& Borut Peterlin ${ }^{2}$ \\ ${ }^{1}$ University Psychiatric Clinic Ljubljana, Ljubljana, Slovenia \\ ${ }^{2}$ Clinical Institute of Medical Genetics, University Medical Centre Ljubljana, Ljubljana, Slovenia
}

received: 25.1.2018; $\quad$ revised: 23.4.2018; $\quad$ accepted: 7.5.2018

\begin{abstract}
SUMMARY
Background: Dementias are clinically and genetically heterogeneous group of neurodegenerative disorders. Often, dementias with genetic etiology are clinically indistinguishable from non-genetic ones. The aim of this retrospective study was to evaluate the yield of clinical exome sequencing in dementias, potentially associated with monogenic genetic predisposition.

Subjects and methods: For this purpose 20 consecutive patients younger than 65 years were studied in the period from January 2014 to December 2017; 14 with the diagnosis of Frontotemporal dementia (FTD), 3 with early-onset Alzheimer disease (EOAD) and 3 with unspecified dementia. In addition to clinical exome sequencing including 57 genes associated with dementia, C9orf 72 hexanucleotide expansion as tested in all patients.

Results: We found genetic etiology in 6 patients: 2 mutations in the PSEN1 gene (p.Pro264Ser and p.Phe105Cys) in the EOAD patients, C9orf72 expansion and MAPT (c.1920+16C>T), mutation in the FTD group of patients as well as MAPT (c.1920+16C >T) mutation and likely pathogenic mutation in the TYROBP mutation (p.Asp32Asn) in patients with unspecified diagnosis.

Conclusions: Our preliminary results imply significant diagnostic yield in identifying rare genetic causes of dementia, combining comprehensive clinical exome sequencing and targeted C9orf72 expansion testing.
\end{abstract}

Key words: dementia - exome sequencing - early-onset dementia - Alzheimer's disease - frontotemporal dementia

$$
* * * * *
$$

\section{INTRODUCTION}

Dementia is a clinical syndrome caused by neurodegeneration which leads to progressive deterioration of cognitive ability (Prince et al. 2013). World Alzheimer Report 2015 estimated that 46.8 million people worldwide had dementia. It is expected that the number will increase to 74.7 million by 2030 and 131.5 million by 2050. Therefore, dementias present increasingly important public health and socioeconomic issue (Wu et al. 2017). Alzheimer's disease (AD), vascular dementia, dementia with Lewy bodies, and frontotemporal dementia (FTD) comprise most common types of dementia, with $\mathrm{AD}$ being most frequent as it accounts for $70 \%$ of all dementia cases (Plassman et al. 2007). Main characteristics of $\mathrm{AD}$ are prominent episodic memory impairment, deficits in visuospatial orientation and apraxia (Apostolova 2016). Frontotemporal dementias are characterized by behavioural changes and/or language deficits, with relatively preserved episodic memory and visuospatial orientation (Rascovsky et al. 2007, Seelaar et al. 2011). Patients with vascular dementia have difficulties with executive functions, while patients with Lewy bodies dementia experience visual hallucinations and parkinsonian motor difficulties (Smits et al. 2015). Cognitive decline trajectories, therefore, differ based on type of dementia, however, symptoms and signs often overlap. This makes the specific diagnosis of dementia, especially early in the development of disease, challenging.
An additional challenge in clinical diagnosis is associated with recognition of monogenic causes of dementia. It was estimated that $25 \%$ of all people aged 50 years and older have a first-degree relative suffering from dementia. Familial aggregation is most commonly associated with genetically complex etiology of disease, where many genetic variations of small effect interact to increase risk of dementia. Only a small proportion of families have an autosomal dominant family history of early-onset dementia, which is often due to Mendelian disease, caused by a mutation in one of the dementia genes (Loy et al. 2014). Nevertheless, early onset dementia is not rare, between 2 and $10 \%$ of all cases of dementia begin before the age of 65 years. Among early-onset dementias, the genetic background of $\mathrm{AD}$ and FTD has been most investigated. Early-onset AD (EOAD) represents $1-6 \%$ all $\mathrm{AD}$ cases and has genetic background in $60 \%$ of cases, $13 \%$ of which is inherited in autosomal dominant fashion (Campion et al. 1999). By definition, disease starts before 65 years of age with similar clinical course as non-genetic $\mathrm{AD}$, although cognitive decline tends to be faster (Mendez 2006, Jacobs et al. 1994). Main genetics risk factors for early-onset mendelian AD are mutations in APP (10-15\% of cases), PSEN1 (20-70\% of cases) and PSEN2 (rare, approximately $5 \%$ of cases) (Campion et al. 1999). FTD is the second most common cause of early-onset dementia (before 65 years of age) (Alzheimer's Association, 2015). A family history of dementia or related neurologic and/or psychiatric illness is present in $40-50 \%$ of 
patients with FTD. 10-30\% of FTD with genetic cause is inherited in autosomal dominant fashion, the rest of the cases present sporadic mutations (See et al. 2010). The majority of Mendelian autosomal dominant cases are caused by mutations in MAPT $(6.3 \%$ of cases $)$, GRN (7.6\% of cases) and C9ORF72 (11.7\% of cases) (DeJesus-Hernandez et al. 2011).

In addition to EOAD and FTD more than 30 monogenic disorders present with or include dementia as a clinical symptom.

Current guidelines for genetic testing of dementias are still based on the Sanger sequencing (Burgunder et al. 2010). For example, European Federation of Neurological recommendation (2010) recommended targeted approach to genetic testing; mutational screening of PSEN1, APP and PSEN2 genes in the case of a clinical diagnosis of $\mathrm{AD}$, and genetic testing for mutations in PRGN and MAPT if the clinical diagnosis is FTLD. However, targeted approaches cannot address the genetic heterogeneity of dementias. New technologies, namely next-generation sequencing (NGS) offer genetic testing of several genes in one genetic test and have potential to substantially improve diagnostic approach.

However, there is still limited evidence of clinical implementation of next-generation sequencing in diagnosis of dementia (Nicolas et al. 2016, Blauwendraat et al. 2018, Sassi et al. 2014). Therefore, we present preliminary study using next-generation exome sequencing on 20 Slovenian patients suffering from different subtypes of dementias.

\section{SUBJETS AND METHODS}

We conducted a retrospective study of consecutive series of patients with dementia tested with clinical exome sequencing at the Clinical Institute of Medical Genetics from January 2014 to December 2017. Criteria for testing were familial or sporadic dementias and included Alzheimer disease, Frontotemporal dementia or unspecified dementia (where clinicians did not come to a specific diagnosis) with the onset at 65 years or younger. Patients received full neurological workup, with neuropsychological examination, magnetic resonance imaging (MRI) and blood analysis. All patients were referred by neurologist or psychiatrist and evaluated by a clinical geneticist. The study was exempted from Institutional Board Review as a retrospective study of clinical practice.

In the studied period, we tested 20 patients, 14 with the diagnosis of FTD, 3 with EOAD and 3 with unspecified of dementia. There were 14 males and 6 females, the average age of symptoms onset was 54.15 years. Six patients had family history of dementia.

Patients were first tested for $\mathrm{C} 9$ orf 72 hexanucleotide expansion and patients with no mutation were further tested by clinical exome sequencing.

Sizing and repeat primed (RP) PCRs for the sizing of alleles and the detection of C9orf72 (G4C2)4 hexanucleotide expansion polymorphism were performed using FAM-labelled primers as previously described (Renton et al. 2011). Final detection and sizing of PCR products was performed on the ABI 3500 Instrument (Applied Biosystems) using GeneScanTM LIZ600 size standard v.2.0. (Applied Biosystems).

CES was performed using the in-solution capture of exonic sequences with Nextera Rapid Capture Enrichment kit (Illumina, USA) targeting the exons of 4813 genes associated with human genetic disease (TruSight One Panel by Illumina, USA). Sequencing was performed on the Illumina MiSeq platform in $2 \times 100$ pair-end reads. Raw sequence files were processed using a custom in-house exome analysis pipeline, based on a GATK best practices backbone. Alignment of reads to the human reference assembly (hg19) was performed using the Burrows-Wheeler (BWA) aligner, duplicate sequences removed using Picard MarkDuplicates, which was followed by base quality score recalibration, variant calling, variant quality score recalibration and variant filtering using elements of the GATK toolset (Alzheimer's Association 2015, Likar et al. 2018).

Variants were stored and annotated in the variant collection and annotation system, based on vtools and ANNOVAR software. Refseq gene models were used for transcript positioning of variants and annotations from dbSNP v138 were used for single nucleotide polymorphism (SNP) annotation. The Slovene genomic variation database, based on a compilation of 2000 exomes was considered the primary source for assessment of variants' prevalence in the population. Furthermore, the datasets of the Exome Aggregation Consortium (ExAC, exac.broadinstitute.org), UK10K control population (www.uk10k.org) and GoNL (www.nlgenome.nl) projects were employed as sources of variant frequencies in other worldwide populations. Consensus calls of dbNSFP v2 precomputed pathogenicity predictions were used for evaluation of pathogenicity for missense variants. Additionally, SNPeff predictors were utilized as a means of parallel annotation of variant effects. GERP++ rejected substation (RS) scores were used as the fundamental information source of evolutionary sequence conservation. Our pipeline included ClinVar, HGMD (http://www.hgmd.cf.ac.uk/ac/index.php) and LOVD (http://www.lovd.nl/3.0/home) as sources of known disease association for identified variants.

Interpretation of sequence variants and reporting of genetic testing results were generally implemented according to the standards and guidelines provided by the American College of Medical Genetics and Genomics and the Association for Molecular Pathology (ACMG) (Tumienè et al. 2017). Specifically, variants were classified as (i) pathogenic; (ii) likely pathogenic, (iii) variants of uncertain significance (VUS), (iv) likely benign, and (v) benign.

All patients were bioinformatically analysed for selected 57 dementia genes: APP, PSEN1, PSEN2, C9ORF72, GRN, MAPT, VCP, TREM2, CHMP2B, CHCHD10, SQSTM1, FUS, TARDBP, CSF1R, TBK1, 
TUBA4A, UBQLN2, HNRNPA2B1, SNCB, SNCA, VPS35, ATP13A2, ITM2B, OPTN, MATR3, DCTN1, TYROBP, NOTCH3, HTRA1, PRNP, DNTM1, TIMM8A, C19ORF12, LRRK2, PINK1, COL4A1, DNMT1, SERPINI1, GLA, GBA1, FMR1, PPT1, HEXA, ATN1, ARSA, CYP27A1, ABCD1, PSAP, GALC, ATXN1, ATXN2, ATXN3, ATXN7, NPC1, NPC2, ATP7B, PANK2 and HTT.

\section{RESULTS}

In patients with FTD, we found C9orf72 expansion in a female patient without family history. Additionally, we found a known MAPT (c.1920+16C $>$ T) mutation in a male patient with positive family history of dementia.

Among EOAD patients we found two previously reported PSEN1 mutations, the p.Pro264Ser mutation in a male with the onset of dementia at 41 years and no family history and the p.Phe105Cys mutation in female patient with the onset of dementia at 45 years with family history of disease. There was the same MAPT $(\mathrm{c} .1920+16 \mathrm{C}>\mathrm{T})$ mutation in a male patient with unspecified dementia and the age of onset of 52 years and no family history.

Finally, a new, likely pathogenic mutation in the TYROBP mutation (p.Asp32Asn) in a patient with unspecified diagnosis, age of onset at 52 years and no family history was found. The variant is predicted to result in a substitution of amino acid aspartate to asparagine at position 32 in TYROBP protein sequence. While the theoretical predictions for this missense variant do not show a consistent pathogenic effect, we observed that it alters the final nucleotide of exon 2 at the intron-exon splice site. We used tools for theoretical predictions of splicing effect (MaxEnt and Human Splice Finder 3.0) to show that this variant (c.94G>A) most likely disrupts the wild-type donor site required for proper splicing of intron 2 from the consensus TYROBP transcript. The rarity of the variant in the general population and high evolutionary conservation of this nucleotide position further supported its likely pathogenic nature.

\section{DISCUSSION}

In this retrospective study, we evaluated the yield of clinical exome sequencing in dementias, primarily dementias potentially associated with monogenic genetic predisposition. We performed NGS sequencing on samples from 20 patients with dementia and found genetic etiology in 6 patients (33\%).

In the FTD group of patients of whom $21 \%$ had a positive family history of dementia, we found C9orf72 expansion in sporadic patient and known pathogenic MAPT $(c .1920+16 C>$ T) mutation in the patient with family history of disease. Our results are comparable with the recent German study (Blauwendraat et al. 2018) in which whole exome sequencing was combined with targeted screening for C9orf72 repeat expansion, GRN and MAPT mutations and cerebrospinal fluid A $\beta 1-42$ and serum progranulin measurements. Namely, in the consecutive series of FTD subjects in which $34 \%$ had a positive family history for neurodegenerative disorder authors reported diagnostic yield of 19\%, mutations in C9orf72 and GRN genes representing 65\% of the molecular pathology.

Among three patients with EOAD in our study, who all had family history of dementia, we found known mutations in PSEN1 gene in two of them. Whole exome sequencing provided diagnostic yield of $22 \%$ in the German cohort of EAOD patients (Blauwendraat et al. $2018)$ and much lower yield (1.5\%) in the French cohort of familial and sporadic EAOD patients (Nicolas et al. 2016). PSEN1 gene mutations were most common in the French population (Nicolas et al. 2016, Lanoiselée et al. 2017), while PSEN2 and APP mutations presented most of the molecular pathology in the German cohort (Blauwendraat et al. 2017).

We have identified a new, likely pathogenic mutation in the TYROBP gene in a patient with no specific diagnosis of dementia. It has been previously suggested that rare variants in the TYROBP gene may be associated with the EOAD (Pottier et al. 2016) but that was not found a common cause of dementia in a Turkish cohort (Darwent et al. 2017). Additionally, the same MAPT mutation was present in a sporadic patient with no specific diagnosis of dementia.

Our study presents the first case of clinical exome sequencing for non-selected dementias in the clinical setting and suggests that clinical exome sequencing, encompassing all known genes associated with monogenic dementias provide a powerful tool in diagnosis of earlyonset dementias. It is especially helpful early in diagnostic process, as clinical symptoms and signs are not specific and therefore specific diagnosis of different types of dementia present a diagnostic challenge.

The limitation of this study is a limited number of patients tested, therefore robust estimates on the molecular pathology of different dementia phenotypes are not possible.

\section{CONCLUSION}

Our preliminary results imply significant diagnostic yield in identifying rare genetic causes of dementia, combining comprehensive clinical exome sequencing and targeted $\mathrm{C} 9$ orf72 expansion testing. Therefore, guidelines for genetic testing approach to monogenic dementias should be modified accordingly.

\section{Acknowledgements: None.}

\section{Conflict of interest: None to declare.}




\section{Contribution of individual authors:}

Bojan Zalar: concept of the article, review manuscript, writing part of manuscript;

Aleš Maver: data analysis;

Ana Kovanda: writing part of the manuscript

Ana Peterlin: prepairing data for analysis, writing part of manuscript;

Peterlin Borut: review manuscript.

\section{References}

1. Alzheimer's Association: Alzheimer's disease facts and figures. Alzheimers Dement 2015; 11:332-84

2. Apostolova LG: Alzheimer disease. Continuum (Minneap Minn) 2016; 22:419-34

3. Blauwendraat C, Wilke C, Simón-Sánchez J, Jansen IE, Reifschneider A, Capell A, et al.: The wide genetic landscape of clinical frontotemporal dementia: systematic combined sequencing of 121 consecutive subjects. Genet Med 2018; 20:240-9

4. Burgunder JM, Finsterer J, Szolnoki Z, Fontaine B, Baets $J$, Van Broeckhoven C, et al.: EFNS guidelines on the molecular diagnosis of channelopathies, epilepsies, migraine, stroke, and dementias: EFNS Guidelines/CME Article. Eur J Neurol 2010; 17:641-8

5. Campion D, Dumanchin C, Hannequin D, Dubois B, Belliard S, Puel M, et al.: Early-onset autosomal dominant Alzheimer disease: prevalence, genetic heterogeneity, and mutation spectrum. Am J Hum Genet 1999; 65:664-70

6. Darwent L, Carmona S, Lohmann E, Guven G, KunRodrigues $C$, Bilgic B, et al.: Mutations in TYROBP are not a common cause of dementia in a Turkish cohort. Neurobiol Aging 2017; 58:240.e1-240.e3

7. DeJesus-Hernandez M, Mackenzie IR, Boeve BF, Boxer AL, Baker M, Rutherford NJ, et al.: Expanded GGGGCC Hexanucleotide Repeat in Noncoding Region of C9ORF72 Causes Chromosome 9p-Linked FTD and ALS. Neuron 2011; 72:245-56

8. Jacobs D, Sano M, Marder K, Bell K, Bylsma F, Lafleche $G$, et al.: Age at onset of Alzheimer's disease: relation to pattern of cognitive dysfunction and rate of decline. Neurology 1994; 44:1215-20

9. Lanoiselée HM, Nicolas G, Wallon D, Rovelet-Lecrux A, Lacour M, Rousseau $S$, et al.: APP, PSEN1, and PSEN2 mutations in early-onset Alzheimer disease: A genetic screening study of familial and sporadic cases. PLoS Med 2017; 14:e1002270

10. Likar T, Hasanhodžić M, Teran N, Maver A, Peterlin B, Writzl K: Diagnostic outcomes of exome sequencing in patients with syndromic or non-syndromic hearing loss. PLoS ONE 2018; 13:1-14
11. Loy CT, Schofield PR, Turner AM, Kwok JBJ: Genetics of dementia. Lancet 2014; 383:828-40

12. Mendez MF: The accurate diagnosis of early-onset dementia. Int J Psychiatry Med 2006; 36:401-12.

13. Nicolas G, Wallon D, Charbonnier C, Quenez O, Rousseau $S$, Richard AC, et al.: Screening of dementia genes by whole-exome sequencing in early-onset Alzheimer disease: Input and lessons. Eur J Hum Genet 2016; 24:710-6

14. Plassman BL, Langa KM, Fisher GG, Heeringa SG, Weir $D R$, Ofstedal MB, et al.: Prevalence of dementia in the United States: The aging, demographics, and memory study. Neuroepidemiology 2007; 29:125-32

15. Pottier C, Ravenscroft TA, Brown PH, Finch NA, Baker $M$, Parsons $M$, et al.: TYROBP genetic variants in earlyonset Alzheimer's disease. Neurobiol Aging 2016; 48:222.e9-222.e15

16. Prince M, Bryce R, Albanese E, Wimo A, Ribeiro W, Ferri $C P$ : The global prevalence of dementia: A systematic review and metaanalysis. Alzheimer's Dement 2013; 9:63-75

17. Rascovsky K, Hodges JR, Kipps CM, Johnson JK, Seeley $W W$, Mendez MF, et al.: Diagnostic Criteria for the Behavioral Variant of Frontotemporal Dementia (bvFTD): Current Limitations and Future Directions. Alzheimer Dis Assoc Disord 2007; 21:S14-8

18. Renton AE, Majounie E, Waite A, Simon Sanchez J, Rollinson S, Gibbs JR, et al: A hexanucleotide repeat expansion in C9ORF72 is the cause of chromosome 9p21linked ALS-FTD. Neuron 2011; 72:257-68

19. Sassi C, Guerreiro R, Gibbs R, Ding J, Lupton MK, Troakes $C$, et al.: Investigating the role of rare coding variability in Mendelian dementia genes (APP, PSEN1, PSEN2, GRN, MAPT, and PRNP) in late-onset Alzheimer's disease. Neurobiol Aging 2014; 35:2881.e12881.e6

20. See TM, Lamarre AK, Lee SE, Miller BL: Genetic causes of frontotemporal degeneration. $J$ Geriatr Psychiatry Neurol 2010; 23:260-8

21. Seelaar H, Rohrer JD, Pijnenburg YAL, Fox NC, Van Swieten JC: Clinical, genetic and pathological heterogeneity of frontotemporal dementia: A review. $J$ Neurol Neurosurg Psychiatry 2011; 82:476-86

22. Smits LL, Van Harten AC, Pijnenburg YAL, Koedam ELGE, Bouwman FH, Sistermans N, et al.: Trajectories of cognitive decline in different types of dementia. Psychol Med 2015; 45:1051-9

23. Tumienè B, Maver A, Writzl K, Hodzić A, Čuturilo G, Kuzmanić-Šamija R, et al.: Diagnostic exome sequencing of syndromic epilepsy patients in clinical practice. Clin Genet 2018; 93:1057-62

24. Wu Y-T, Beiser AS, Breteler MMB, Fratiglioni L, Helmer $C$, Hendrie HC, et al.: The changing prevalence and incidence of dementia over time - current evidence. Nat Rev Neurol 2017; 13:327-39

Correspondence:

Prof. Bojan Zalar, MD, PhD

University Psychiatric Clinic Ljubljana

Studenec 48, 1260 Ljubljana, Slovenia

E-mail:bojan.zalar@guest.arnes.si 